\title{
The Milky Way Halo and the First Stars: New Frontiers in Galactic Archaeology
}

\author{
Timothy C. Beers ${ }^{1,2}$, Jason Tumlinson ${ }^{3}$, Brian O'Shea ${ }^{1,2}$, Carolyn \\ Peruta $^{1,2}$ Daniela Carollo ${ }^{4,5}$ \\ ${ }^{1}$ Department of Physics \& Astronomy, Michigan State University, \\ email: beers@pa.msu.edu \\ ${ }^{2}$ Joint Institute for Nuclear Astrophysics \\ ${ }^{3}$ Space Telescope Science Institute \\ ${ }^{4}$ Research School of Astronomy \& Astrophysics, ANU, Australia \\ ${ }^{5}$ INAF, Osservatorio Astronomico di Torino
}

\begin{abstract}
We discuss plans for a new joint effort between observers and theorists to understand the formation of the Milky Way halo back to the first epochs of chemical evolution. New models based on high-resolution N-body simulations coupled to simple models of Galactic chemical evolution show that surviving stars from the epoch of the first galaxies remain in the Milky Way today and should bear the nucleosynthetic imprint of the first stars. We investigate the key physical influences on the formation of stars in the first galaxies and how they appear today, including the relationship between cosmic reionization and surviving Milky Way stars. These models also provide a physically motivated picture of the formation of the Milky Ways "outer halo," which has been identified from recent large samples of stars from SDSS. The next steps are to use these models to guide rigorous gas simulations of Milky Way formation, including its disk, and to gradually build up the fully detailed theoretical "Virtual Galaxy" that is demanded by the coming generation of massive Galactic stellar surveys.
\end{abstract}

Keywords. astronomical data bases: surveys, Galaxy: halo, structure, methods: data analysis, n-body simulations, stars: abundances

The explosion of detailed astrometric, photometric, and spectroscopic data for stars in the Milky Way (and Local Group galaxies) that is coming upon us both now (e.g., SDSS/SEGUE, RAVE), and in the near future (PanSTARRS, SkyMapper, Gaia, LSST, SIM Lite) will fundamentally change our vision of galaxy formation and evolution. Full exploitation of this wealth of new information requires the development of sophisticated numerical models capable of producing testable predictions against which the observations can be compared. We have initiated one such effort, foreshadowed by the work of Tumlinson (2006), and continued by Tumlinson (2009). The new models predict the locations, kinematics, and chemistry of the stars in the Galactic halos, with different assumptions concerning the nature of the first stars and their effects on subsequent stellar generations. We are also exploring new methods for visualizing both the predictions and the existing databases. The first efforts will compare expectations with observed differences in the inner- and outer-halo populations, e.g., as in Carollo et al. (2007).

\section{References}

Carollo, D., et al. 2007, Nature, 450, 1020

Tumlinson, J. 2006, ApJ, 641, 1

Tumlinson, J. 2009, ApJ, submitted 